
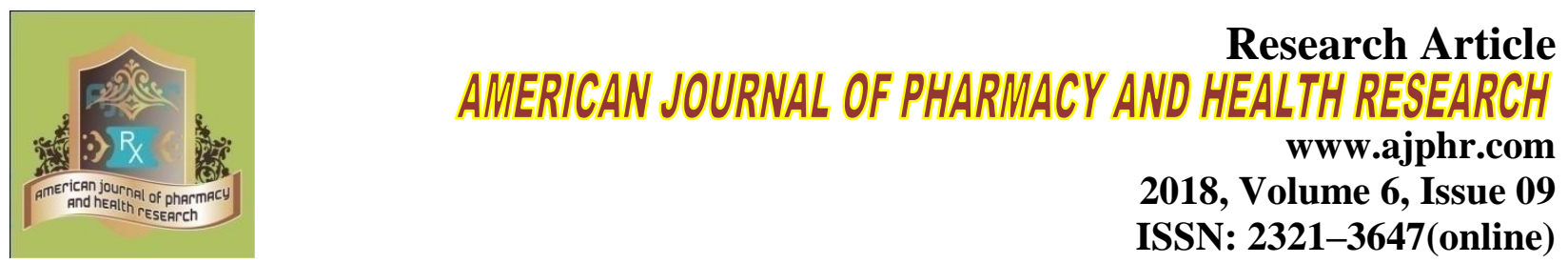

2018, Volume 6, Issue 09

ISSN: 2321-3647(online)

\title{
Studies on the Antimicrobial Activity of Ethanolic Extract of Whole Plant of Saccharum Spontaneum (Linn.)
}

\author{
J. Amutha Iswarya Devi*1, K. Madhumitha ${ }^{1}$, N. Venkateshan ${ }^{1}$ \\ 1 Arulmigu Kalasalingam College of Pharmacy, Anand Nagar, Krishnankoil-626 126, \\ Tamil Nadu, India.
}

\begin{abstract}
In recent years, there has been a growing interest in researching and developing new antimicrobial agents from various sources to combat microbial resistance. Therefore, a greater attention has been paid to antimicrobial activity screening and evaluating methods. The research work was conducted to investigate the antimicrobial activity of ethanolic extract of whole plant of Saccharum spontaneum Linn. (Family: Poaceae). Disc diffusion technique was used for antibacterial and cup plate method was used for antifungal screening. Antibacterial tests were performed by disc diffusion method on nutrient agar, in order to analyze the percentage zone of inhibition. Whole plant's extract showed the significant zone of inhibition (mm), against Staphylococcus aureus, Bacillus subtilis, Escherichia coli and Pseudomonas aeruginosa. Modified agar well diffusion method was used to measure the minimum inhibitory concentration (MIC). Antifungal method was performed by cup plate method against Candida albicans. Due to presence of tannins, polyphenolic compounds and flavonoids, it inhibits the growth of bacteria on most regulatory levels.
\end{abstract}

Keywords: Saccharum spontaneum, ethanolic crude extract, agar diffusion method, cup plate method. 


\section{INTRODUCTION}

Traditional use of medicinal plants and its products have a long history that began with folk medicine and through the years has been incorporated into allopathic medicine (Dubey et al., 2011). Since antiquity, many plants species reported to have pharmacological properties as they are known to possess various secondary metabolites like flavonoids, glycosides, alkaloids, saponins, steroids, tannins, terpenes. Over $50 \%$ of all modern clinical drugs are of natural product origin and natural products play an important role in drug development programs in the pharmaceutical industry (Backer et al., 1995). Antimicrobials of plant origin have enormous therapeutic potential. They are effective in the treatment of infectious diseases while simultaneously mitigating many of the side effects that are often associated with synthetic antimicrobials. Herbal drugs have gained importance in recent years because of their efficacy and cost effectiveness.

Saccharum spontaneum linn (family - poaceae) is a tall erect reed like perennial grass with plume like inflorescence, grows in marshes areas. Leaves and stalks contain lignin, carbohydrates, proteins and amino acids. Roots and root-stocks contain starch and polyphenolic compounds. Aerial parts possess laxative and aphrodisiac properties and are useful in burning sensations, strangury, phthisis, vesicles calculi, blood diseases, biliousness and haemorrhagic diathesis (Chopra et al., 1992). Roots are used as galactagogue and diuretic. The present study was undertaken to investigate the antimicrobial activity of the ethanolic extractives of Saccharum spontaneum.

\section{MATERIALS AND METHOD}

\section{Plant material :}

The plant of saccharum spontaneum was collected from Shengottai, Thirunelveli district, Tamilnadu.

\section{Plant material extraction :}

The plants were collected, dried under shade and triturate into coarse powder material. They were extracted in soxhelt apparatus in a round bottom flask with ethanol at $40^{\circ} \mathrm{C}$ for three days. Then the extract was separated. The extract was stored in amber coloured glass air-tight container. Then the crude extract was ready for assaying of antimicrobial activity.

The percentage yield was calculated for the extracts and major compounds with reference to the crude material taken using the formula given below. 


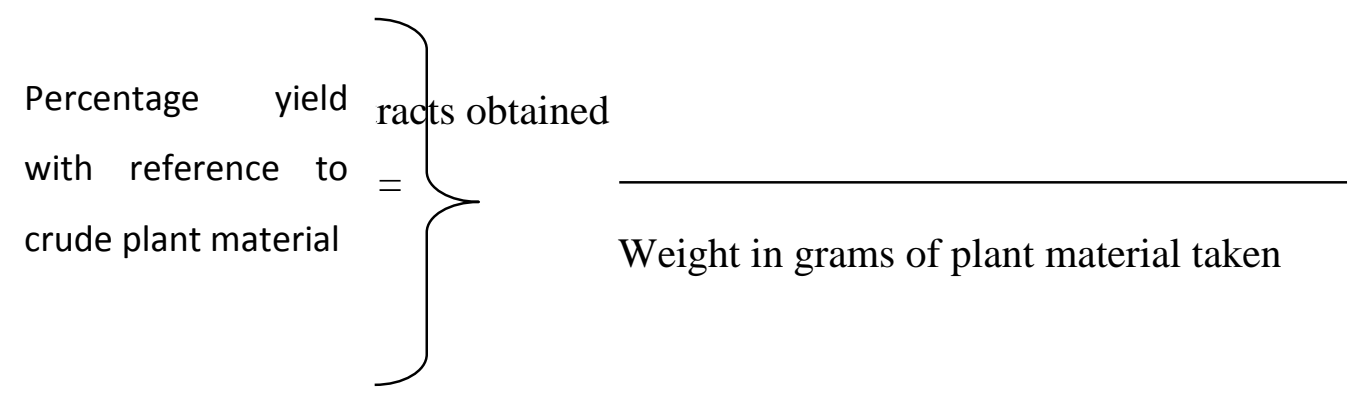

Table 1 Percentage yield of the whole plant of Saccharum spontaneum

\begin{tabular}{llllc}
\hline Plant name & Parts used & Method of extraction & Solvent system & $\begin{array}{l}\text { Percentage yield } \\
(\boldsymbol{\%} \mathbf{w} / \mathbf{w})\end{array}$ \\
\hline $\begin{array}{l}\text { Saccharum } \\
\text { spontaneum }\end{array}$ & Whole plant & $\begin{array}{l}\text { Continuous hot } \\
\text { percolation }\end{array}$ & Ethanol & 8.47 \\
\hline
\end{tabular}

\section{Test organism and standard drugs used:}

Standard drugs such as ciprofloxacin and norfloxacin are used. Whereas test micro organism such as Bacillus subtilis, Staphylococcus aureus, Pseudomonas aeruginosa and Escherichia coli were collected. All microbes were cultured overnight in a nutrient agar pH 5 containing peptone $(0.5 \%)$, agar $(1.2 \%)$, yeast $(0.3 \%)$ and $\mathrm{NaCl}(0.8 \%)$.

\section{Antimicrobial test:}

Antimicrobial assay was performed by adopting the standard disc diffusion method. Two types of discs were used i.e., standard discs (ciprofloxacin inhibit the DNA synthesis and norfloxacin inhibit the bacterial cell wall biosynthesis) and crude extract discs (sample dics). All the discs have diameter of $6 \mathrm{~mm}$. Glass wares and prepared nutrient agar media were sterilized in autoclave at $121^{\circ} \mathrm{C}$ for 25 minutes. Agar plates were prepared with thickness of gel layer ranging between $2-3 \mathrm{~mm}$. The petri dishes were incubated overnight at $37^{\circ} \mathrm{C}$ to allow the bacterial growth. The anti microbacterial activities of the extracts were then determined by measuring the respective zone of inhibition in $\mathrm{mm}$.

\section{Antifungal screening:}

Antifungal activity of ethanolic extract of whole plant of Saccharum spontaneum Linn. was determined against pathogenic fungi (candida albicans). Ketaconazole was used as standard.

\section{RESULTS AND DISCUSSION}

\section{Antibacterial activity:}

The screening and evaluation of antibacterial activity was carried out by agar disk diffusion method. The agar disk-diffusion method is not appropriate to determine the minimum inhibitory concentration (MIC), as it is impossible to quantify the amount of the antimicrobial agent 
diffused into the agar medium. Which was carried out by using different concentrations. The test microorganisms are gram positive microorganism (Bacillus subtilis and Staphylococcus aureus) and gram negative bacteria (E.coli and Pseudomonas aeruginosa).

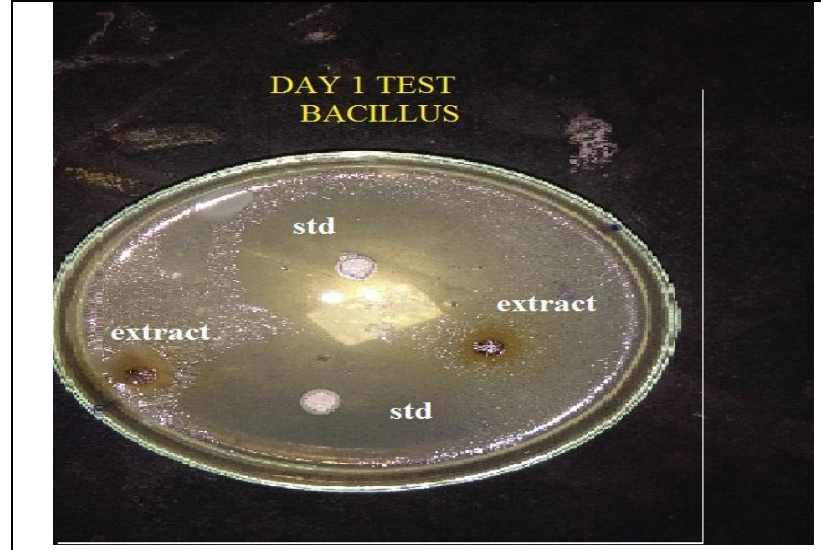

Figure: 1 Effect of antimicrobial activity in Bacillus subtilis

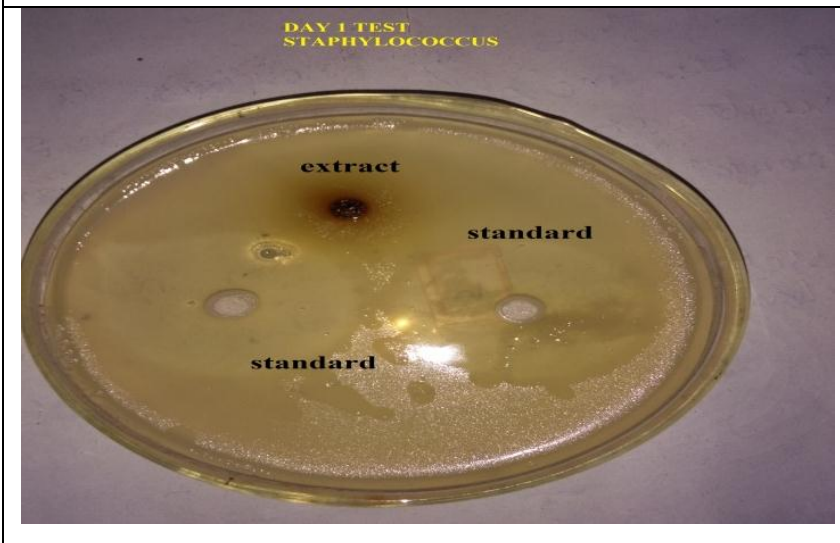

Figure.:3 Effect of antimicrobial activity in Staphylococcus aureus

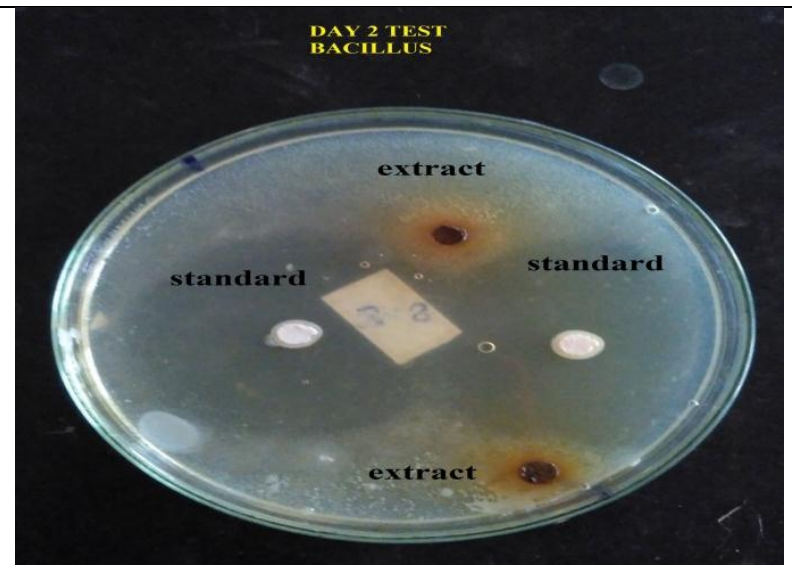

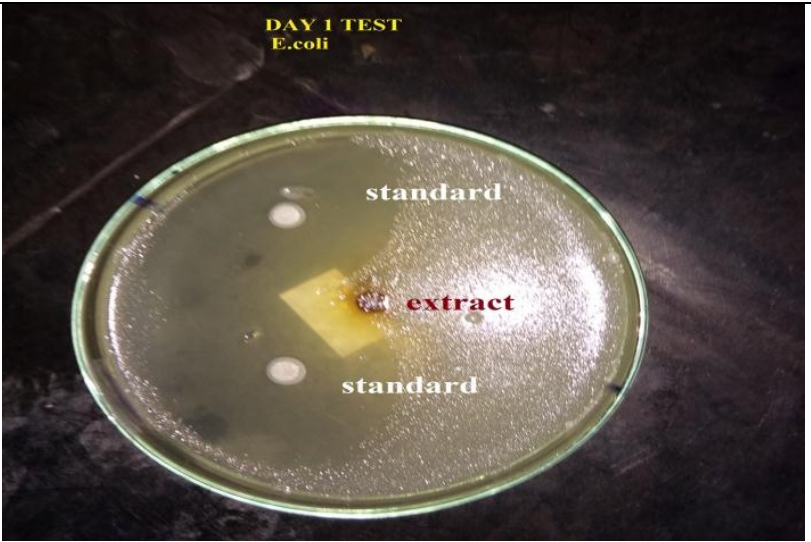

Figure:2 Effect of antimicrobial activity in E.coli

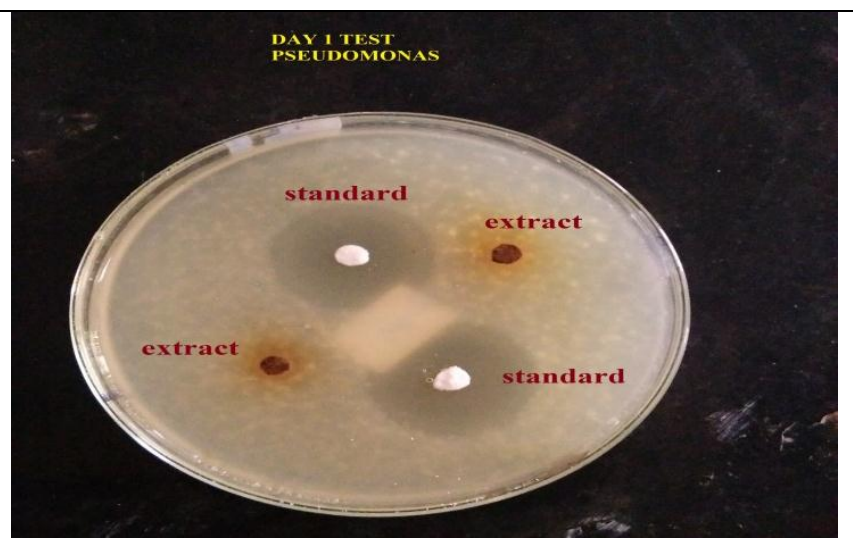

Figure.:4 Effect of antimicrobial activity in Pseudomonas aeruginosa






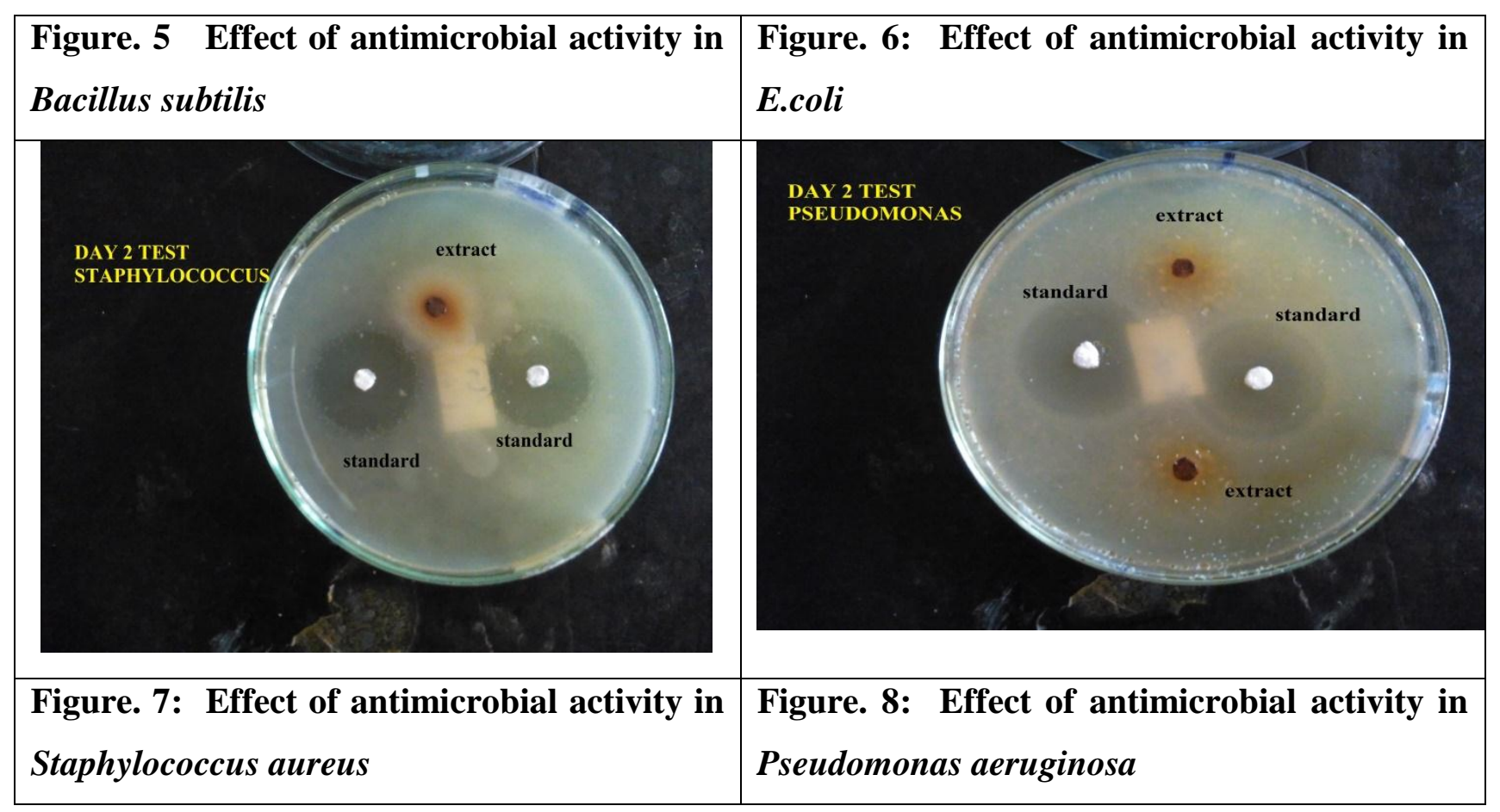

Table 2 Effect of antimicrobial activity of ethanolic extract of S. spontaneum

\begin{tabular}{llllll}
\hline Petri dish & Microorganism & \multicolumn{2}{c}{ Day 1 test } & \multicolumn{2}{c}{ Day 2 test } \\
\cline { 3 - 6 } & & Zone of inhibition ( diameter) & \\
\cline { 3 - 6 } & & Standard & Extract & Standard & Extract \\
\hline 1 & Bacillus & $3.5 \mathrm{~cm}$ & $1.0 \mathrm{~cm}$ & $4.2 \mathrm{~cm}$ & $1.3 \mathrm{~cm}$ \\
2 & Bacillus & $2.4 \mathrm{~cm}$ & $1.0 \mathrm{~cm}$ & $2.8 \mathrm{~cm}$ & $1.2 \mathrm{~cm}$ \\
3 & E.coli & $3.4 \mathrm{~cm}$ & $0.8 \mathrm{~cm}$ & $4.5 \mathrm{~cm}$ & $1.2 \mathrm{~cm}$ \\
4 & E.coli & $3.5 \mathrm{~cm}$ & $1.3 \mathrm{~cm}$ & $3.6 \mathrm{~cm}$ & $1.3 \mathrm{~cm}$ \\
5 & Staphylococcus & $4.0 \mathrm{~cm}$ & $1.4 \mathrm{~cm}$ & $4.0 \mathrm{~cm}$ & $1.4 \mathrm{~cm}$ \\
6 & Staphylococcus & $2.3 \mathrm{~cm}$ & $0.9 \mathrm{~cm}$ & $2.5 \mathrm{~cm}$ & $1.2 \mathrm{~cm}$ \\
7 & Pseudomonas & $2.5 \mathrm{~cm}$ & $1.0 \mathrm{~cm}$ & $2.7 \mathrm{~cm}$ & $1.2 \mathrm{~cm}$ \\
8 & Pseudomonas & $3.4 \mathrm{~cm}$ & $1.2 \mathrm{~cm}$ & $3.5 \mathrm{~cm}$ & $1.2 \mathrm{~cm}$ \\
\hline
\end{tabular}




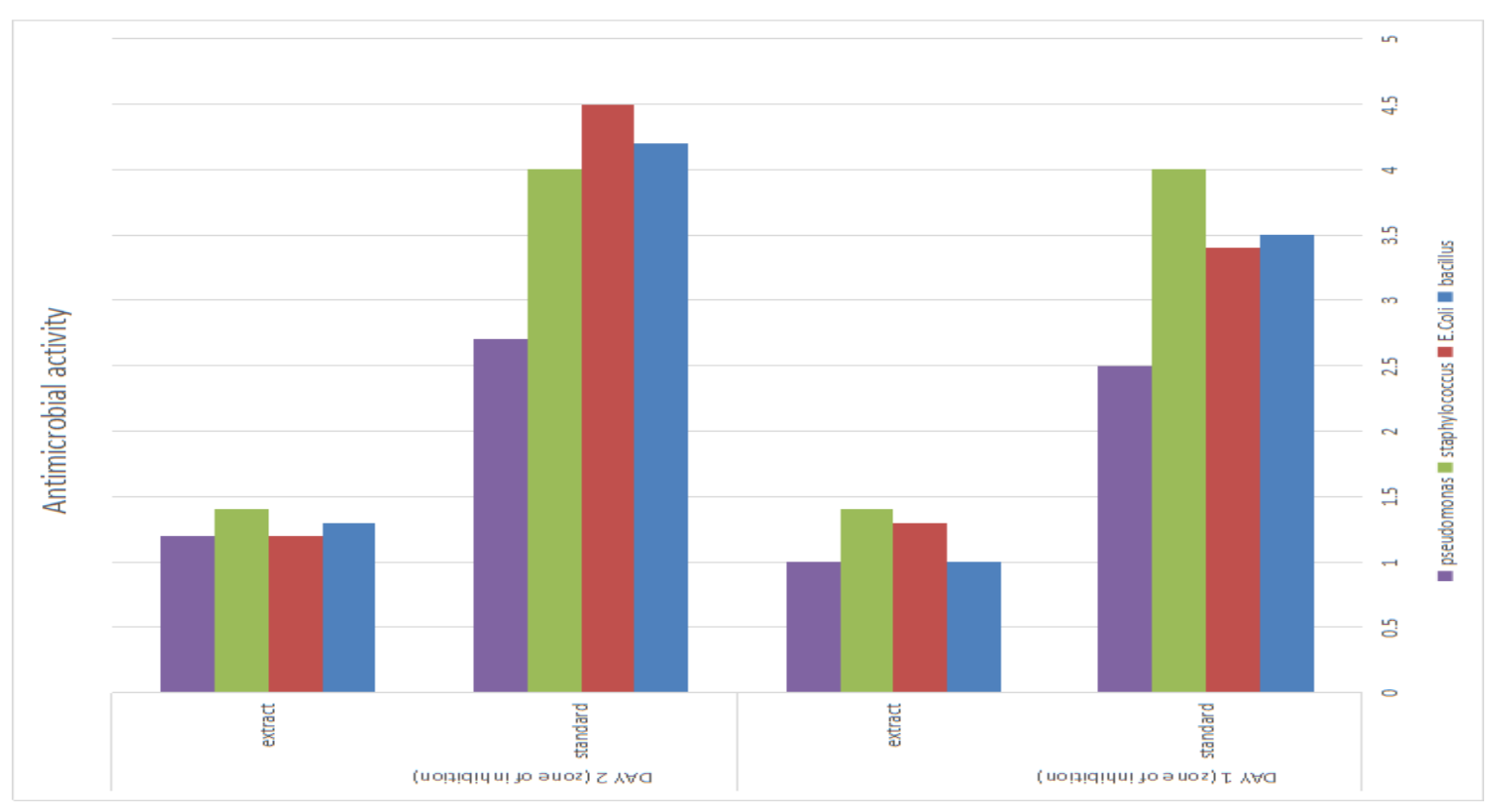

Figure 1 Effect of antimicrobial activity of ethanolic extract of S.spontaneum ANTIFUNGAL ACTIVITY:

The screening and evaluation of antifungal activity was carried out by agar well diffusion method and determination of MIC values, which was carried out by using different concentration. The test fungus was Candida albicans.

\section{Results of determination of MIC value}

After evaluating the ethanolic extract of S. spontaneum was taken which shows higher antifungal activity for MIC test by taking a different concentration. The test fungus was inoculated in different concentration of plant extracts i.e. $10 \mu \mathrm{L}, 20 \mu \mathrm{L}, \ldots . .100 \mu \mathrm{L}$.

\begin{tabular}{llllllllllll}
\hline Extract & \multicolumn{1}{c}{ Different volume of plant extracts $(\mu \mathrm{L})$} \\
\cline { 2 - 9 } & $\mathbf{1 0}$ & $\mathbf{2 0}$ & $\mathbf{3 0}$ & $\mathbf{4 0}$ & $\mathbf{5 0}$ & $\mathbf{6 0}$ & $\mathbf{7 0}$ & $\mathbf{8 0}$ & $\mathbf{9 0}$ & $\mathbf{1 0 0}$ \\
\hline S.spontaneum & + & + & + & + & + & + & - & - & - & - \\
\hline
\end{tabular}

C. albicans was strongly inhibited by the ethanolic extract of whole plant of S. spontaneum. This suggests that plant extract can be used to inhibit the growth of $C$. albicans and thus they can be implicated in the prevention and treatment of oral candidal infections. The efficacy of plant and their extract was due to the presence of several primary and/or secondary metabolites such as phenolics, polyphenols, tannins, quercetin, flavones, flavonols, alkloids, terpenoids, and complex mixtures. Although phytochemicals (plant derived metabolites) are antimicrobial in nature but they also produce other biological activities in the oral cavity like induction of immunity, which indirectly reduces the risk of oral diseases. 


\section{REFERENCE}

1. Sathya M, Kokilavani R Antiurolithiatic activity of ethanolic root extract of Saccharum spontaneum on glycolic acid induced urolithiasis in rats. Journal of Drug Delivery \& Therapeutics, 2012, 2(5), 86-89.

2. Amutha Iswarya Devi $\mathrm{J}$ and A. Kottai Muthu. Evaluation of hypolipidemic activity of ethanolic extract from whole plant of Saccharum spontaneum Linn. in rat fed with atherogenic diet, Der Pharmacia Lettre, 2015; 7 (8):103-109.

3. Amutha Iswarya Devi $\mathrm{J}$ and A. Kottai Muthu. Evaluation of in-vitro free radical scavenging potential of whole plant of Saccharum spontaneum (Linn). International Journal of Pharm Tech Research,2014; 6(5): 1436-1440.

4. Amutha Iswarya Devi J and A. Kottai Muthu. Gas chromatography-mass spectrometry analysis of bioactive constituents in the ethanolic extract of Saccharum spontaneum Linn. International Journal of Pharmacy and Pharmaceutical Sciences,2014; 6(2): 755-759.

5. Amutha Iswarya Devi J and A. Kottai Muthu. Phytochemical screening, antioxidant activities and total phenolic content of ethanolic extract from whole plant of Saccharum spontaneum (Linn.), International Journal of Chemical and Pharmaceutical Sciences, 2014; 5 (2): 112-118.

6. Mayers DL, Lerner S A and Ouelette M et al. Antimicrobial Resistance in staphylococcus aureus mechanisms of resistance, Clinical and Epidemiological Aspects, 2009; 2: 681-1347.

7. Harborne JB Phytochemical methods 11 Edn. In Chapman \& Hall. NewYork.1984: 4-5.

8. Jeyaraman Amutha Iswarya Devi and A. Kottai Muthu Isolation and characterization of active components derived from whole plant of Saccharum spontaneum(Linn.), Der Pharmacia Lettre, 2015; 7 (8): 197-203.

9. Mohammad Khalid, Hefazat H. Siddiqui Free radical scavenging and total phenolic content of saccharum spontaneum .IJRPC 2011, 1(4)

10. Mohammad Khalid, Hefazat H. Siddiqui pharmacognostical evaluation and qualitative analysis of saccharum spontaneum B (linn.) root international journal of pharmaceutical sciences and drug research,2011(b) 3(4), 338-341.

11. Mynol Islam Vhuiyan MD, Israt Jahan Biva, Moni Rani Saha, Muhammad Shahidul Islam. Anti-diarrhoeal and CNS Depressant Activity of Methanolic Extract of Saccharum spontaneum Linn. Stamford Journal of Pharmaceutical Sciences,2008; 1(1\&2): 63-68. 
12. Sai Krishna M, Naga Sravanthi K, Sreenika G, Chaithanya S, Sudhakar M.In vitro antioxidant activity of methanolic extract of Saccharum spontaneum, Int. J. Pharm. 2013; 3(3): 628-633.

13. Sathya $M$ and Kokilavani R. Acute and sub acute toxicological assessment of the ethanolic root extract of Saccharum spontaneum Linn. (Poaceae) in male wistar albino rats, Journal of Chemical and Pharmaceutical Research, 2012; 4(8): 3894-3899.

AJPHR is

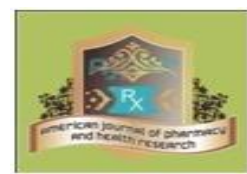

Peer-reviewed

monthly

Rapid publication

Submit your next manuscript at editor@ajphr.com / editor.ajphr@gmail.com 